\title{
Los sistemas expertos y la recuperación documental: ejemplos de aplicación
}

\author{
Pedro Manuel Aguado \\ Profesor Titular de Matemática Aplicada \\ Centro Politécnico Superior \\ Universidad de Zaragoza
}

\subsection{Resumen}

Las Nuevas Tecnologías de la Información y las Comunicaciones han proporcionado nuevas técnicas en la obtención de fuentes de información. La posibilidad de acceso a grandes bases de datos tanto específicas como multidisciplinares ha hecho de la búsqueda de información automatizada, una práctica habitual. Por ello, los Sistemas de Información deben ser creados, tomando como meta la utilización y difusión de la información contenida en ellos y pensando en la conveniencia de que dicha utilización sea realizada por el usuario final. Por esta razón el diseño y creación de herramientas que hagan fácil y agradable la consulta de información es una línea de desarrollo en pleno auge. Son descritos casos, en los cuales se pone claramente de manifiesto, la vigencia de la construcción de estos interfaces, como son proyectos de investigación de la Comunidad Europea o la publicación de leyes que regulan y fomentan el acceso del ciudadano a la documentación pública. En el trabajo se estudian distintos tipos de interfaces de usuario que corresponden a tres niveles diferentes: a) interfaces tipo recordar y teclear, como son los lenguajes de interrogación y recuperación documental; b) interfaces tipo ver y hacer, basados en una metodología de construcción de "menús "; y c) interfaces tipo hablar, oír y pedir, construidos en base a sistemas expertos. Se dan ejemplos concretos de los tipos mencionados, en los cuales ha participado el autor del trabajo y se presenta la metodología desarrollada en cada uno de éstos. De todo ello se deduce que la utilización de la técnica de la Inteligencia Artificial posibilita la creación de estas herramientas de modo que tanto su utilización, como los resultados obtenidos, son satisfactorios tanto para el profesional que las desarrolla, como para el usuario final que las necesita, personaliza y utiliza. (Autor)

Palabras clave: Sistemas Expertos, Recuperación Documental, Interfaces, 
Inteligencia Artificial.

\subsection{Abstract}

The new information and communication technologies have provided with new techniques in information sources retrieval. The possibility to access to great databases - both specific and multidisciplinary - has transformed automated information retrieval a normal practice. That is why information systems must be created with the aim of the use and dissemination of the information stored in them, and having in mind the final user's access as a very convenient goal. So, the design and implementation of tools to make easier information retrieval is a thriving research line. Several of such systems are described, as research projects of the European Union or in the field of public administration citizen's information. The interfaces are classified into three levels: remind and type (e. g., retrieval languages), take a glance and do (menus), and talk, listen and ask, based on expert systems. Some examples of each category developed by the author of the paper are explained, together with their design and implementation methodology. These experiences demonstrate that using Artificial Intelligence tools and techniques results in the satisfaction of both the developer and the user.

Keywords: Expert systems, Information Retrieval, Interfaces, Artifical Intelligence

\section{Introducción.}

Actualmente estamos inmersos en una sociedad en la cual se está produciendo no ya la revolución de la informática, sino la revolución de la Información, y ello está suponiendo cambios profundos y radicales en la búsqueda de información previa necesaria tanto para la investigación como para la toma de decisiones.

Así, es conocida y aceptada la gran importancia, aún más, de la necesidad de estar bien informado.

Ningún profesional está ajeno a los avances que las Nuevas Tecnologías de la Información están suponiendo, y no es exagerado afirmar que, en estos momentos, ninguna Institución, equipo de investigadores, estudioso o postgraduado comenzará una línea de investigación, tesis o trabajo de investigación en general, sin antes haber efectuado las correspondientes consultas a las bases de datos adecuadas.

Han evolucionado pues y mucho, todos los aspectos relacionados con este tema y han nacido nuevas vías y posibilidades que todo profesional debe conocer y ser conocedor de la información, que en un campo determinado, está a su alcance, contenida en las bases de datos tanto nacionales como internacionales.

Scire. $1: 2$ (jul.-dic. 1995). 
Estas bases de datos pueden estar soportadas ya sea en sistemas on-line, o bien mediante las técnicas de CD-Rom, pero en cualquier caso es necesario conocer su utilización y funcionamiento para poder extraer de ellas la información correcta, de una forma eficaz y exhaustiva.

La evolución indicada está produciendo importantes cambios en los sistemas de tratamiento y conservación de la información debidos, fundamentalmente, a los avances logrados, en los últimos años, en dos campos concretos: las redes de telecomunicación y la microelectrónica, produciendo ordenadores cada vez más potentes y baratos. Para definir esa imbricación de las telecomunicaciones y de la informática, los especialistas han inventado el término "telemática" que ya empieza a ser incorporado en los manuales escolares.

Los efectos más tangibles de esta imbricación, que ya comienza a observar con una cierta curiosidad y preocupación el ciudadano medio, se sitúan en la eclosión de nuevos servicios, a los cuales se puede acceder desde el propio hogar, a través de un terminal y de la línea telefónica: el acceso a los bancos de datos, la telecompra, el correo electrónico, la tele-conferencia, etc.

Asistimos, pues, a un cambio importante, que nos va a exigir a todos dar una respuesta positiva al desafío electrónico, como la dio la sociedad en el momento del descubrimiento de la máquina de vapor, del ferrocarril y de la electricidad. Porque, a pesar de los posibles riesgos, la telemática tiene muchos factores positivos, si sabemos aprovechar al máximo sus grandes posibilidades.

Al mismo tiempo, la posibilidad de acceso a esa ingente cantidad de información hace aparecer unos problemas específicos nuevos no conocidos hasta ahora.

De todos ellos, sería digno de destacar lo que se podría denominar exceso de oferta de información, lo explicamos:

El problema actual consiste no tanto en hallar información pertinente sino en cómo examinar si la información obtenida es la mejor posible y sobre todo, saber si es toda la existente, en un determinado tema específico.

Por todo ello se hace necesario un completo conocimiento de las bases de datos existentes en el campo elegido, tanto a nivel nacional como internacional.

Pero no sólo es necesario conocer esta existencia de las bases de datos sino que hay que conocer cómo es su protocolo de conexión y sobre todo y fundamental, conocer su lenguaje de consulta. Desde este punto de vista, el panorama es complejo, pues por una parte se tiene la posibilidad de acceso a gran cantidad de bases de datos, mediante técnicas de conexión sencillas y por otra se tiene la existencia de gran cantidad de lenguajes de recuperación de información distintos entre sí. 
Este aspecto apuntado es importante, pues a la hora de efectuar una búsqueda de información, el conocimiento del lenguaje de recuperación, a un nivel lo más profundo posible, es fundamental. Teóricamente cuando se conecta con una base de datos se dispone de toda la información contenida en ella pero, sin embargo, hay que saber encontrarla, seleccionarla y obtenerla.

Se hace pues necesaria la colaboración de perfiles profesionales distintos. Pues no hay que olvidar que para poder consultar una base de datos, primero hay que crearla, diseñarla y mantenerla. Para ello se habrá de contar con el equipo de especialistas concretos del tema a tratar, pero además se habrá de contar con el informático documental.

El equipo de especialistas estudiará qué información se debe introducir en la base de datos. El informático documental decidirá el cómo, y todo ello pensando en la recuperación de esa información introducida. Más adelante se volverá sobre este importante aspecto.

Otro paso más avanzado sería el que el usuario de esa información, esto es, el usuario final, pudiera obtener la información deseada de forma fácil preguntándola en un lenguaje normal, no informático, esto es, en lenguaje natural. En este sentido la técnica de creación de interfaces combinada con la utilización de la Inteligencia Artificial puede ayudarnos a obtener soluciones.

Por ello, seguidamente se trata de exponer de una forma breve y concisa las distintas posibilidades, que en estos momentos se poseen, de acceso a la documentación, teniendo siempre como objetivo que las técnicas utilizadas hagan la consulta fácil y agradable al usuario que la efectúa, sin que éste posea especiales conocimientos ni de Informática ni de Documentación.

Es de destacar que la creación y utilización de este tipo de técnicas hará posible la difusión de las posibilidades y ventajas documentales que poseen, acercando la consulta automatizada de información a colectivos cuya utilización de este tipo de consulta no ha sido, en general, muy habitual.

Por otra parte, parece claro que el objetivo a conseguir en este campo, es el garantizar el mejor nivel posible de información a todos los organismos públicos, empresas privadas y ciudadanos en general, para que todos puedan desarrollar sus actividades profesionales y personales con la mejor base informativa posible.

Además, toda técnica encaminada a un tratamiento, cada vez más depurado de la información es una meta importante, pues es cierta una frase utilizada con frecuencia en la literatura especializada: Las personas actúan sobre la información y la información actúa sobre las personas.

Se expone seguidamente una afirmación:

El éxito de un sistema de información no depende exclusivamente de las

Scire. 1 : 2 (jul.-dic. 1995). 
soluciones técnicas aportadas sino que está condicionado en gran medida a la reacción y acogida entre el personal técnico, informático y documental que lo mantiene y los usuarios que lo consultan.

La idea expresada es, entendemos, importante pues, en general, las personas que diseñan un sistema de información lo hacen pensando y confiando que tal sistema será utilizado por el usuario final y satisfará sus problemas de información en un campo determinado.

Lamentablemente en muchos casos esta suposición no llega a cumplirse, debido, en parte, a que para su manejo se hace necesaria, cuando no imprescindible, la presencia de un intermediario especialista por no estar el sistema suficientemente orientado hacia el usuario final.

Esta situación obliga, entonces, a que el usuario posea conocimientos, cuando menos, a buen nivel tanto de informática como de documentación.

Todas las afirmaciones anteriores están basadas en la experiencia informática y documental del autor del presente trabajo, obtenida mediante la creación del denominado Centro de Documentación Científica, de la Universidad de Zaragoza, para lo cual hubo de trabajar tanto en la elaboración de bases de datos propias, como en la extracción y explotación de la información de bases de otros productores, tanto a nivel nacional como internacional.

Resaltaremos pues dos hechos que deberían ser tenidos presentes por los creadores, tanto de bases de datos como de sistemas de información:

$1^{\circ}$ ) Las bases de datos deben ser creadas y elaboradas teniendo como meta la explotación de la información contenida en ellas.

$2^{\circ}$ ) Que la información contenida en ellas, esté recogida de modo que pueda ser explotada por el usuario final.

Sin embargo:

- esta explotación por el usuario final no es siempre fácil y accesible,

- existen gran cantidad de lenguajes de recuperación distintos entre sí,

- los protocolos de conexión no siempre son satisfactorios.

Por todo ello se han trabajado y estudiado distintos aspectos, tanto desde el punto de vista informático y de las comunicaciones, como desde la perspectiva de la documentación, pensando siempre para ello en el usuario final y en la recuperación de información. Por esta razón nos centraremos en lo que se podría denominar "interfaces de usuario" y las posibilidades y herramientas de que en estos momentos disponemos, entre ellas, los sistemas expertos.

La creación de estos interfaces, tiene una doble dimensión: técnica y social.

Dimensión técnica, pues trata de investigar y mejorar las herramientas de que

Scire. $1: 2$ (jul.-dic. 1995). 
actualmente se dispone, logrando una mayor adaptabilidad al usuario final.

Dimensión social, ya que una gran parte de estos interfaces de usuario son utilizados para suplir discapacidades de la persona y tienen como objetivo la integración de esas personas en el entorno social habitual en donde tienen que vivir.

Para tratar de ser exhaustivos, consideraremos varios niveles, que se especifican a continuación.

\section{Primer nivel: Lenguajes de recuperación documental}

En este nivel se situarían los lenguajes de recuperación documental cada vez más perfectos y flexibles como son QUEST, GRIPS, etc. y sobre todo DIALOG. Estos lenguajes, si bien no son difíciles de manejar, su utilización es, en general, compleja para un usuario no especialista en este tipo de lenguajes.

Por ello tras los estudios y experiencias realizadas, se ha obtenido como conclusión importante que el proceso de consulta debe ser transparente para el usuario que la realiza, en el sentido de que el proceso de consulta sea siempre el mismo, sin que sea afectado por el ordenador en el que se efectúa la consulta o el lenguaje de recuperación utilizado.

\section{Segundo nivel: Interfaces basados en menús}

La solución dada por este nivel al proceso de transparencia mencionado, estaría constituida por la construcción de interfaces que, sin ser todavía inteligentes, proporcionan al usuario una importante ayuda a la consulta de información.

Los interfaces, en este nivel, están basados en la elaboración de menús construidos de tal forma que, una vez efectuada la elección por el usuario se ejecutan una serie de órdenes y programas en el ordenador, totalmente transparentes para el usuario y que dan como resultado el objetivo deseado, cual es, la búsqueda de la información deseada.

A la espera de los anunciados interfaces inteligentes, en lenguaje natural, están resucitando, de nuevo, los sistemas basados en menús, si bien se puede decir que no son los menús rígidos de hace unos pocos años, sino que se presentan como un interface amigable de usuario que permite, por lo general, personalizarlos en función de opciones previas determinadas por éste. En este sentido, el usuario puede elegir el idioma y la temática de consulta, el rango de bases de datos a consultar o incluso el tipo de menú, bien en forma de textos o de iconos, mediante utilidades gráficas. Esto es, se ha pasado de interfaces en los cuales había que "recordar y teclear", a interfaces en los cuales la clave es "ver y hacer".

Con la utilización de este tipo de menús se fomenta y facilita la búsqueda de información por parte del usuario final que puede no ser un especialista en bús-

Scire. $1: 2$ (jul.-dic. 1995). 
queda documental, ni conocer los lenguajes de recuperación específicos.

En este sentido, el autor ha experimentado este tipo de interfaces, creando con herramientas informáticas estándar, una serie de pantallas intermedias interactivas generadas de forma automatizada por el ordenador y que mediante subrutinas internas, muestran al usuario cómo debe efectuar la búsqueda de información de acuerdo con sus necesidades y obviando de este modo el conocimiento del lenguaje documental utilizado.

Dicha experiencia está operativa con un ordenador IBM 4381 y con herramientas propias de este entorno informático: el lenguaje de recuperación documental STAIRS y el paquete ISPF.

Este interface, si bien no es inteligente, es capaz de reconocer y validar la información pedida, informando al usuario de algún posible error cometido en el rango de su consulta y mostrando mediante mensajes tanto el error como la posible solución. Pero, lo importante a resaltar es que dichas pantallas o menús de ayuda están vinculadas directamente con el lenguaje documental utilizado de modo que una vez cumplimentadas éstas, se efectúa la búsqueda de información como si los comandos del lenguaje hubieran sido tecleados, aprovechando por tanto toda la potencia y recursos que el lenguaje nos ofrece. La metodología seguida es extrapolable y portable a otros entornos informáticos.

Es de hacer notar, por otra parte, la posibilidad de efectuar la búsqueda de información utilizando los comandos convencionales del lenguaje, caso de que el usuario lo desee, pues existe una pantalla que permite, bien esta posibilidad, bien la utilización de las pantallas mencionadas.

Otro ejemplo de este tipo de interfaces que podemos citar, es el desarrollado por uno de los grupos de investigación del Departamento de Matemática Aplicada del Centro Politécnico Superior de Ingenieros, de la Universidad de Zaragoza, y al cual el autor de este artículo pertenece, mediante el cual se ha implementado un programa, que optimiza un problema real, de una gran empresa, como es la carga masiva de mercancía, de acuerdo con un gran número de parámetros, altamente restrictivos, como son, por ejemplo: dimensiones, pesos, fragilidad, capacidad y ruta de los camiones o containers a cargar, criterios económicos específicos, etc.

Pero no solo se ha optimizado esa carga, sino que una vez realizado ese cálculo, de acuerdo con los datos aportados y mediante un interface combinando gráficos, iconos y colores, la solución es presentada de una forma clara y nítida, fácilmente interpretable por cualquier persona no especialista en el tema, la cual puede ver sin ningún esfuerzo cómo ha de distribuir la carga. Así pues, en este nivel, todavía no se está utilizando sistemas inteligentes, pero se está haciendo un uso inteligente de los sistemas.

Scire. 1 : 2 (jul.-dic. 1995). 


\section{8}

Estas metodologías descritas, podrían ser aplicables para resolver lo expresado en el Acta Final del Tratado de Maastrich, la cual pone de manifiesto el derecho de acceso a la documentación de la administración pública, lo cual además de favorecer el control democrático de la administración, obliga a organizar la documentación para que ésta sea fácilmente localizable.

Asimismo, sería aplicable al ejemplo concreto de la Ley de Régimen Jurídico de las Administraciones Públicas y del Procedimiento Administrativo común, de España, publicada en el Boletín Oficial del Estado de 27 de Noviembre de 1992 y que ha entrado en vigor el 27 de Febrero de 1993.

Dicha ley tiene como objetivos, entre otros, la agilización de los trámites burocráticos, la simplificación del sistema de recursos administrativos y la utilización de las nuevas tecnologías informáticas.

Estos objetivos tienen unas claras consecuencias en las relaciones de la administración con los ciudadanos, quienes se hacen acreedores de derechos tales como la posibilidad de conocer el estado de la tramitación de los procedimientos administrativos relacionados con éstos, o el acceso a registros y archivos que contengan información pública.

Es de destacar, que dicha ley reconoce la validez de los documentos emitidos y recibidos por medios informáticos o telemáticos, lo cual supondrá una mayor simplificación y un ahorro de tiempo considerable.

Sin embargo, somos conscientes de que el sistema de menús puede tener inconvenientes, sobre todo si las opciones de éstos son muy numerosas y por tanto la utilización del interface dejaría de ser de utilización "agradable" que es justamente lo que se quiere conseguir. Por ello seguidamente se considerará el:

\section{Tercer nivel: Sistemas expertos}

Aquí se considerará la utilización de herramientas inteligentes como ayuda al usuario. Este tercer nivel de creación de interfaces de usuario de búsqueda de información tiene plena vigencia, como es puesto de manifiesto, en los proyectos de demostración piloto, pertenecientes al programa IMPACT I de la Comunidad Europea, y en los que los conceptos de interfaces de información independientes del dominio, técnicas multimedia, información técnica específica y sistemas expertos aplicados a la documentación se entrelazan entre sí.

El objetivo de que cualquier diálogo que se pueda establecer con el ordenador sea más grato y que pueda eliminarse el sistema de menús, se consigue mediante la elaboración de programas, capaces de detectar las intenciones del usuario. De aquí la necesidad de posibilitar un diálogo inteligente con el ordenador.

Scire. $1: 2$ (jul.-dic. 1995). 
Así, la consecución de una información determinada de cualquier tipo y en cualquier materia, cuyos parámetros y condiciones de búsqueda estén perfectamente definidos, no requerirá seguramente la utilización de ninguna herramienta inteligente.

Sin embargo, la búsqueda de una información en la cual las palabras clave o los datos que se tienen, o no son suficientes o no están bien definidos, necesitará la utilización de programas de búsqueda que interactúen de forma inteligente con el usuario para que éste obtenga el dato o información que pretende.

Por otra parte, la utilización del conocimiento por usuarios finales, especializados en un tema específico pero no habituados al uso de lenguajes formalizados, nos lleva de nuevo a la idea ya expuesta de transparencia para el usuario pues esa utilización necesitará del desarrollo de estrategias conducentes a un rápido, eficaz y selectivo manejo de la información contenida en la base de conocimientos. Pero dicho proceso debería efectuarse sin que el usuario final sepa cómo trabaja internamente el sistema, ni que tareas se están ejecutando.

Además, es interesante la idea de lograr como objetivo que la creación de esas estrategias o programas especiales sea, utilizando como herramientas de creación, técnicas o programas estándares. Por otra parte, la creación de estos programas que llamaremos "interfaces inteligentes ", inciden no solo en la consulta y la utilización "amigable" de la información sino que hacen posible la captura del conocimiento proporcionado por el especialista (que jugaría el papel de ingeniero de conocimiento en el tema de que se tratase), para que dicho conocimiento sea almacenado y tratado en el ordenador.

Todo lo expresado anteriormente, es lo que se ha desarrollado en la Universidad de Zaragoza por parte de un grupo interdisciplinar en el cual el autor de este trabajo ha participado como investigador y dentro del cual se ha creado un prototipo cuya breve descripción se dará al final del trabajo, basado y construido por medio de una shell y en el cual tanto la base de datos como el programa que vincula al experto y a la base de datos han sido construidos en lenguaje Pascal.

Estamos pues ante la creación de sistemas expertos y esta potente herramienta requiere su propia metodología, pues los sistemas expertos difieren de las aplicaciones tradicionales en muchos aspectos entre los cuales se pueden destacar:

- La gran sofisticación y depuración del interface de usuario.

- La existencia de una base de conocimientos, o sistema de conceptos relacionados que permiten al ordenador aproximarse al razonamiento humano.

- La shell o intérprete. La shell es quién procesa realmente la información introducida por el usuario, la relaciona con los conceptos contenidos en la base de conocimientos y proporciona una conclusión, juicio o solución a 
un problema formulado o a una pregunta realizada.

La utilización, pues, de los sistemas expertos presenta una serie de ventajas que pueden resumirse en que:

- Ayudan a responder a preguntas cuyas soluciones son ya conocidas y que están previamente almacenadas en el ordenador.

- Responden a preguntas cuyas soluciones, si bien, no están almacenadas, sí que se pueden inferir de la información ya existente en la base de datos creada y que utiliza como herramienta el sistema experto.

- Justifican todos los razonamientos efectuados en base a la información previa existente.

- Tienen capacidad de aprender tanto de sus propios razonamientos efectuados como de nuevas situaciones que se le planteen e introduzcan, ampliando de este modo la base de conocimientos.

Por otra parte, el desarrollo y utilización de sistemas expertos significa un avance importante en los procesos de recuperación de información. En este sentido es de hacer notar que los principales distribuidores de bases de datos "online" ofrecen la posibilidad de consultar su información mediante la utilización de sistemas expertos específicos, relacionados con información, bien con productos y técnicas informáticas bien sobre Compañías y Empresas, con respecto de informaciones y noticias aparecidas en los principales periódicos de economía a nivel mundial.

En este sentido, la utilización de sistemas expertos en recuperación de información pueden traducirse en las siguientes ventajas:

- Ejercen, como ya se ha visto, un papel de interface entre el usuario final y la base de datos; creando modelos de búsqueda de acuerdo con unos parámetros definidos y manipulando protocolos tanto de conexión como de consulta.

- Conocen cuántas y cuáles son las bases de datos a consultar en función del tema a consultar y de la profundidad de la búsqueda.

- Optimizan los resultados de las búsquedas mediante la utilización de reglas confeccionadas por especialistas específicos del tema de que se trate.

- Utilizan el lenguaje natural para las consultas, con la cual hacen fácil y agradable la consulta de información eliminando la rigidez del lenguaje informático.

- De acuerdo con los resultados obtenidos son capaces de depurar la documentación obtenida, al objeto de redefinir los parámetros originados para obtener un mejor perfil de búsqueda. 


\section{Ejemplos de aplicación del tercer nivel}

Como ya se ha mencionado, se tiene un ejemplo de todo lo expresado con anterioridad desarrollado por un grupo interdisciplinar, en un campo no habitualmente elegido y tan complejo como es el terreno del Derecho.

El ejemplo a describir sigue los tres niveles expresados, puesto que:

- Se ha creado una base de datos conteniendo el Derecho Civil de Aragón. Dicha base puede ser consultada mediante los comandos de un lenguaje de recuperación documental como es el STAIRS. Estamos pues en el primer nivel expresado.

- Posteriormente ha sido creado un interface de usuario, a base de menús, para que esta base pueda ser consultada directamente sin conocer los comandos del lenguaje de recuperación. Dicho interface ha sido construido mediante la utilización del paquete ISPF. Estamos pues en el segundo nivel mencionado.

- Actualmente nos encontramos en el tercer nivel pues se ha trabajado en la construcción del denominado sistema SIREDOJ (Programa de Ayuda a la decisión jurídica), que es un sistema inteligente de recuperación de documentación jurídica.

Los objetivos de la construcción de este prototipo han sido tres: en primer lugar probar la versatilidad y el manejo de una shell estándar, utlizándola como desarrollo de un sistema experto en el terreno del derecho.

En segundo lugar, diseñar y construir una base de datos documental de ámbito jurídico ajustada a las necesidades del problema a tratar y por último lograr que la comunicación del programa con el usuario fuese lo más sencillo posible, atendiendo tanto al lenguaje utilizado, como a la metodología de introducción de información y sobre todo la presentación al jurista como usuario final del sistema.

El prototipo construido ayuda a un abogado a averiguar por medio de un diálogo si para el caso que plantea al ordenador es posible ejercitar una determinada acción judicial, cuál es dicha acción, el procedimiento a seguir y la documentación jurídica que cabe utilizar para fundamentar dichas actividades.

Es un programa que accede pues, a un sistema de recuperación de documentación jurídica, textos legales, referencias jurisprudenciales y referencias bibliográficas.

Es de hacer notar, finalmente, que entendemos que la metodología de trabajo utilizada es extrapolable a otros campos del saber y para otras aplicaciones muy distintas de la mencionada, como podría ser un tema que está en estudio sobre los parques naturales y mejores rutas a seguir en ellos atendiendo a una serie de pará-

Scire. $1: 2$ (jul.-dic. 1995). 
metros que el usuario del sistema de información exige, como pueden ser, especies animales o vegetales a visitar y de cuya existencia se le ha informado previamente, principales características de éstas, tiempo que se desea invertir en el recorrido, etc.

Asimismo, en el Centro de Ingenieros citado anteriormente y dentro de la investigación de un proyecto de la Comunidad Europea, se han desarrollado otro tipo de interfaces pertenecientes a la tercera generación cuyas funciones son "hablar, oír y pedir".

Son pues, interfaces basados en reconocimiento y síntesis de voz y aplicados a personas discapacitadas, con problemas de motilidad, para que éstas puedan controlar su entorno físico y puedan, mediante la utilización de la voz, abrir una puerta, encender una luz, controlar la posición de la cama, o bien utilizar una cocina o una lavadora, a personas con problemas de visión, sin que éstas corran ningún riesgo. Todo ello contribuye a la creación de un denominado "hogar inteligente".

Por todo lo expuesto, creemos que la creación de todo tipo de herramientas inteligentes que puedan servir como ayuda al usuario final, es un tema que debe seguirse y promocionarse tanto a nivel de investigación como de desarrollo.

\section{Referencias}

Aguado, P. M. El Acceso a la Documentación : Creación de Interfaces. // Jornadas Informatización en la Administración Pública. 1989. Vol. 1. p. 9-16.

Bares, M. La Búsqueda Documental en el Contexto Telemático. Diaz de Santos, 1988. $338 \mathrm{p}$.

Benchimol, G. ; Levine, P. ; Pomerol, J. Los Sistemas Expertos en la Empresa. Madrid : Rama. 1988. 199 p.

Buchanan, B. G. ; Shortlifte, E. H. Rule-Based Expert Systems. Addison-Wesley, 1985

Cuena, J. Inteligencia Artificial : Sistemas Expertos. Alianza. 1986. 157 p.

Cood, P. ; Yourdan, E. Object-Oriented Analysis. Prentice-Hall, 1990.

Frost, R. Bases de Datos y Sistemas Expertos : Ingeniería del Conocimiento. Díaz de Santos. 1989. 769 p.

FUINCA. Inteligencia Artificial para la Gestión de Bases de Datos. Madrid : Consejo Superior de Investigaciones Científicas. 1987. 99 p.

Harmon, P.; King, D. Sistemas Expertos. John Wiley \& Sons Inc., 1985.

Hughes, J. G. Object-Oriented Database. Prentice Hall, 1991.

Schaub, A. Sistemas Expertos. // PC World. (Sept. 1989) 154-156.

Scott, A. C. ; Clayton, J. E.; Gibs, E. L. A practical guide to knowledge adquisition. Addison-Wesley, 1991.

Sullivan, J. W. ; Tyler, S. W. Intelligent User Interfaces. Addison-Wesley, 1991.

Scire. 1 : 2 (jul.-dic. 1995). 